\section{ORIGLAR COWTANS \\ COLOR LLUSTRATIOHS \\ 2}

\section{Science}

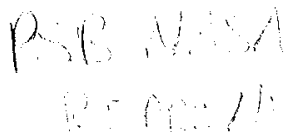

$(6)$

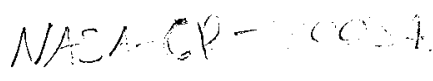

\title{
Spatial Organization and Time Dependence of Jupiter's Tropospheric Temperatures, 1980-1993
}

\author{
Glenn S. Orton, A. James Friedson, \\ Padmavati A. Yanamandra-Fisher, John Caldwell, \\ Heidi B. Hammel, Kevin H. Baines, Jay T. Bergstralh, \\ Terry Z. Martin, Robert A. West, Glenn J. Veeder Jr., \\ David K. Lynch, Ray Russell, Michael E. Malcom, \\ William F. Golisch, David M. Griep, Charles D. Kaminski, \\ Alan T. Tokunaga, Thomas Herbst, Mark Shure
}




\title{
Spatial Organization and Time Dependence of Jupiter's Tropospheric Temperatures, 1980-1993
}

\author{
Glenn S. Orton, A. James Friedson, \\ Padmavati A. Yanamandra-Fisher, John Caldwell, \\ Heidi B. Hammel, Kevin H. Baines, Jay T. Bergstralh, \\ Terry Z. Martin, Robert A. West, Glenn J. Veeder Jr., \\ David K. Lynch, Ray Russell, Michael E. Malcom, \\ William F. Golisch, David M. Griep, Charles D. Kaminski, \\ Alan T. Tokunaga, Thomas Herbst, Mark Shure
}

The spatial organization and time dependence of Jupiter's temperatures near 250-millibar pressure were measured through a jovian year by imaging thermal emission at $18 \mathrm{mi}-$ crometers. The temperature field is influenced by seasonal radiative forcing, and its banded organization is closely correlated with the visible cloud field. Evidence was found for a quasi-periodic oscillation of temperatures in the Equatorial Zone, a correlation between tropospheric and stratospheric waves in the North Equatorial Belt, and slowly moving thermal features in the North and South Equatorial Belts. There appears to be no common relation between temporal changes of temperature and changes in the visual albedo of the various axisymmetric bands.

Planetary atmospheres are characterized in a fundamental way by their temperature structures that govern dynamical forces, reflect the influences of radiative and other sources of heating and cooling, and establish the context in which gaseous chemistry and cloud microphysics take place. We describe the first results of a study of the spatial morphology of Jupiter's temperatures through a jovian year. This work probes a region where energy transfer is influenced by convection as well as radiation, deeper in the atmosphere than addressed in our study of stratospheric temperatures (1). The global and long-term nature of our results is a major extension of previous studies of Jupiter's tropospheric temperatures and dynamics, which have largely been derived from short-duration spacecraft studies (2).

G. S. Orton, A. J. Friedson, K. H. Baines, T. Z. Martin R. A. West, P. A Yanamandra-Fisher are at M.S. 169-237, and G. J. Veeder Jr. is at M.S. 183-501, Jet Propulsion Laboratory, California Institute of Technology, Pasadena, CA 91109, USA; J. Caldwell is at the Space Astrophysics Laboratory, Institute for Space and Terrestrial Science, Concord, Ontario L4K $3 \mathrm{C} 8$ Canada: H. B. Hammel is at the Department of Earth Atmospheric and Planetary Sciences, Building 54 416. Massachusetts Institute of Technology, Cambridge, MA 02139, USA; J. T. Bergstralh is at Code SL-4, National Aeronautics and Space Administration, Washington, DC 20546, USA: M. E. Malcom is at TRW R2/2186, 1 Space Park, Redondo Beach, CA 90278 USA; D. K. Lynch and R. Russell are at the Space Sciences Laboratory, M2-266, The Aerospace Corporation, P.O. Box 92957, Los Angeles, CA 90009, USA W. F. Golisch, D. M. Griep, C. D. Kaminski, A. T. Tokunaga, and $M$. Shure are at the Institute for Astronomy, University of Hawaii, Honolulu, HI 96822 , USA: T. Herbst is at the Max-Planck-Institute für Astronomie, Heidelberg, Germany.
We observed Jupiter between 1980 and 1993 at the NASA/Infrared Telescope Facility on Mauna at Kea, Hawaii (Table 1). Tropospheric temperatures were determined from thermal infrared emission observed by means of a facility photometer with a discrete filter centered at $18.20 \mu \mathrm{m}$ $\left(549.4 \mathrm{~cm}^{-1}\right)$ (3). Near $18 \mu \mathrm{m}$, Jupiter's atmospheric opacity is dominated by the collision-induced absorption of $\mathrm{H}_{2}$ (4) and is minimally influenced by clouds. As $\mathrm{H}_{2}$ is uniformly mixed throughout the atmosphere, the substantial temporal and spatial variations of the thermal emission observed with this filter (Fig. 1) indicate variations of atmospheric temperature.

Central meridian scans obtained in 1980 through 1982 were sampled at 1 arc sec intervals. Partial coverage of Jupiter's disk followed in raster scans made in 1983 and full coverage thereafter, sampling at 1 arc sec intervals in right ascension and declination. The diameter of the circular aperture used in 1980 and 1981 was 3.92 arc sec. For all later observations, the diameter was $1.96 \mathrm{arc} \mathrm{sec}$, close to the diffraction limit, corresponding to $5^{\circ}$ or $6600 \mathrm{~km}$ at the equator. The assignment of latitude, longitude, and emission angle to each pixel of an image required accounting for the drift of the telescope from Jupiter's actual motion in the sky and its rotation during the approximately 20 to 45 minutes required to scan the disk. Spatial uncertainties are on the order of 1 arc sec or less. Our study includes only locations on the disk with emission angles of $56^{\circ}$ or less, as positions closer to the planetary limb suffered perceptibly from blending with cold space.

Just as with our 7.8- $\mu \mathrm{m}$ images (1), we derived absolute radiances by normalizing the mean value observed in the inner 50 percent of a strip along Jupiter's central meridian to the average radiance observed by the Voyager 1 IRIS central meridian maps in a simulated response to the $18-\mu \mathrm{m}$ filter. The net effect minimized temperature changes with time for the largest number of points on the planet, rendering the data insensitive to changes of the global mean temperature, but preserving relative differences. The best images have a signal-to-noise ratio of about 400 (equivalent to an uncertainty of just under $0.05 \mathrm{~K}$ in brightness temperature). Noisier images resulted from poor seeing or from local humidity variations which map the variable signal onto the observed disk as a false spatial variation. The signal-to-noise ratio for most of the images is closer to $100(0.15 \mathrm{~K}$ in brightness temperature). We did not use images with signal-to-noise ratios less than 70 .

In order to interpret the observed radiance in terms of physical temperature, we assumed an average vertical temperature structure. A smoothed average of Voyager 1 radio occultation experiment results (5) was used in an initial guess of the temperature profile; the overlying stratospheric temperatures were cooled, however, (Fig. 2) in order to provide the best fit to the radiances observed across the disk at all emission angles. The resulting profile is close to that of the Voyager radio occultation egress profile (5). This profile was perturbed by the same temperature at all levels among the family of profiles illustrated in Fig. 2 until the observed radiance was matched; this provided a one-to-one correspondence between a temperature profile and the intensity at each pixel of an image. While temperature perturbations that decrease slightly with depth might correspond more closely to Voyager results (5), the differences between the temperatures derived from such perturbations and the simple scheme adopted here are not significant. For locations observed at more than one emission angle, the temperature was derived from the observation at the smallest emission angle, or, in some cases, from the least noisy map. Temperatures are reported at 250 mbar, near the peak of the contribution functions for nadir and limb observations (Fig. 2). While this simple procedure is insensitive to possible variations of the vertical temperature profile from those shown in Fig. 2, such variations are not expected, as the different radio occultation profiles show strikingly similar morphologies near 250 mbar.

Zonal mean thermal structure. At any given latitude, Jupiter exhibits significant temporal and spatial variation of its zonal 
mean (longitudinally averaged) thermal structure. Selected profiles of the zonal mean 250-mbar temperature between 1978 and 1993, together with profiles of the vertical shear of the zonal mean wind are shown in Fig. 3. Most of these profiles were constructed from separate images (Table 1) acquired within 3 days and covering a large longitude range, although some epochs have large gaps in temporal and longitudinal coverage. The temperature profile for 1979 was derived by simulating the $18-\mu \mathrm{m}$ filter response to Voyager IRIS north-south map sequence spectra. The profile shown for 1978 was derived from the $18-\mu \mathrm{m}$ central meridian scan of Sinton et al. (6). The shear was derived from the temperature profile using the thermal wind equation,

$$
f \frac{\partial u}{\partial z}=-\frac{R}{a H} \frac{\partial T}{\partial \phi}
$$

where $u$ is the eastward zonal mean wind, $T$ is the temperature, $z$ is minus scale height times log of the pressure and is used as a vertical coordinate, $\phi$ is latitude and $f, a$, $H$, and $R$ are the Coriolis parameter, planetary radius, pressure scale height, and gas constant, respectively. The geostrophic approximation on which Eq. 1 is based breaks down near the equator where $f$ becomes small, but because Jupiter is a rapidly rotat-

Table 1. Observational summary.

\begin{tabular}{lc}
\hline \multicolumn{1}{c}{ Dates (UT) } & Scans (no.) \\
\hline 1978 February, from (6) & 2 \\
1980 January & 2 \\
1981 March & 2 \\
1982 February & 2 \\
\multicolumn{1}{c}{ Dates (UT) } & Maps (no.) \\
\hline 1983 July 15, 17 & 2 \\
1984 June 6, 8 & 2 \\
1984 July 23 & 2 \\
1984 August 9, 10 & 2 \\
1984 September 10 & 2 \\
1985 May 21 & 1 \\
1985 September 5 & 1 \\
1986 June 6 & 1 \\
1986 July 9 & 1 \\
1987 June 7, 8 & 1 \\
1987 October 25, 26, 27 & 2 \\
1987 December 30 & 4 \\
1988 September 25, 26, 28 November 30 & 2 \\
December 1, 2 & 4 \\
1989 December 15, 16 & \\
1990 January 9 & 3 \\
1990 February 11 & 5 \\
1990 April 6 & 1 \\
1991 February 9, 10 & 1 \\
1991 April 2 & 1 \\
1991 May 5 & 3 \\
1992 October 26, 27 & 1 \\
1993 March 1 1993 March 30, 31 & 1 \\
1993 April 27 & 2 \\
\hline
\end{tabular}

ing planet with zonal winds that are steady over one rotation period, we expect the equation to yield accurate wind shears to within a few degrees of the equator. We estimated the zonal wind shear at $0^{\circ}$ latitude from the formula $H \partial u / \partial z=-[R /$ $(2 \Omega a)] \partial^{2} T / \partial \phi^{2}$ (the planetary rotation period is $2 \pi / \Omega$ ), which strictly is valid only in the case when the temperature profile is symmetric about the equator.

The time-averaged temperature and vertical wind shear profiles reinforce the interpretation of the IRIS data (7). The North and South Equatorial Belts (NEB and SEB) near $\pm 12^{\circ}$ are some 2 to $3 \mathrm{~K}$ warmer than the Equatorial Zone (EZ) and mid-latitude zones. As found by Gierasch et al. (7), the vertical shear of zonal wind tends to be anti-correlated with the zonal jets at the

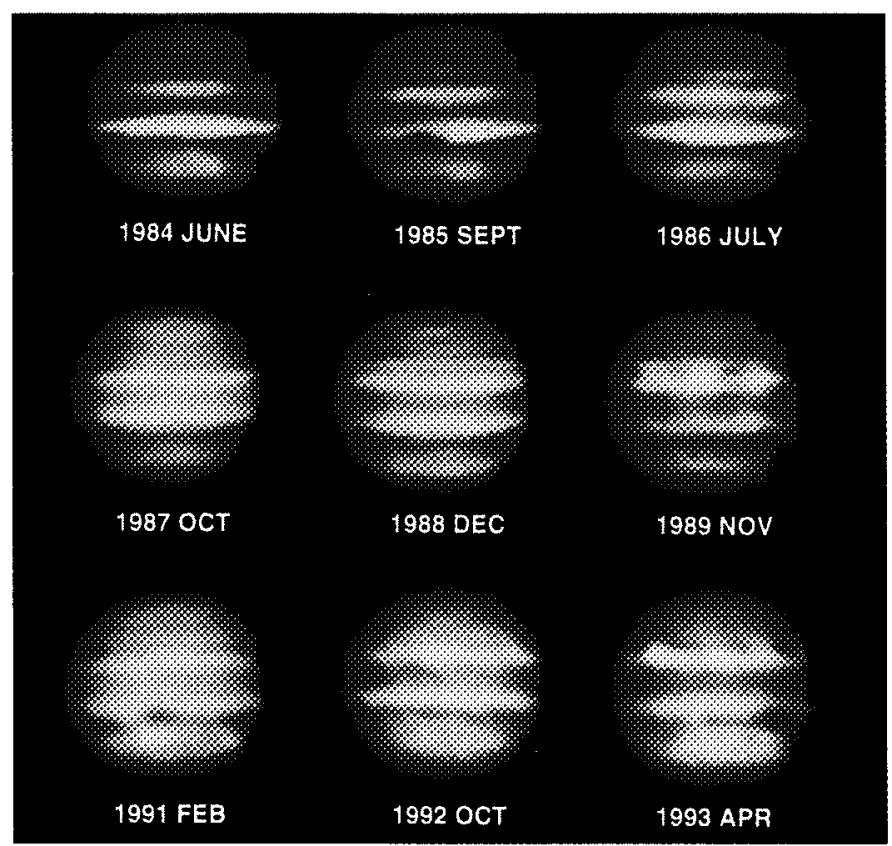

Fig. 1. Raster-scan images of Jupiter, representing the best of the images available for each apparition since 1984, identified by the epoch of observation. Detailed observation dates are given in Table 1 . The warmest regions in each image are shown in white and the coolest in dark red.

Fig. 2. The end members and center of a family of temperature profiles used to generate a table relating outgoing intensity as a function of emission angle with the temperature at a given atmospheric level. These have a cooler stratosphere than a nominal profile, shown by the dashed curve, which represents a mean of Voyager 1 radio occultation experiment results (5). Also shown as dotted curves are the level by level contributions to the outgoing thermal intensity as a function of height for the baseline temperature profile for the 18- $\mu \mathrm{m}$ filter at

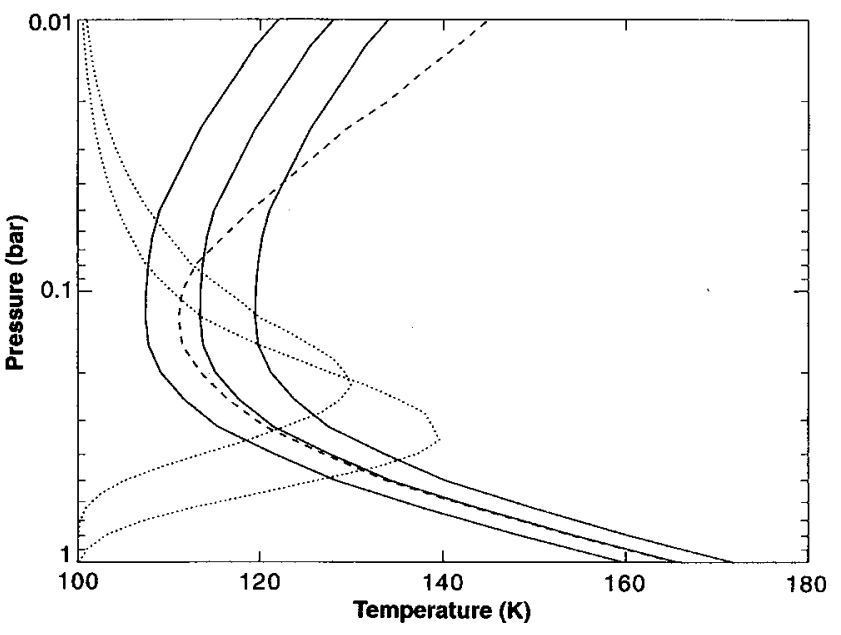
the nadir (lower curve) and at an emission angle of $56^{\circ}$ (upper curve). The contribution functions have arbitrarily normalized amplitudes but are correct relative to one another. cloud-top level, implying a decay of zonal

in Fig. 3 consists of (i) a seasonal variation, (ii) a quasi-periodic oscillation at the equator, and (iii) strong variation of tempera-
tures and vertical wind shears in the latitude band 20 to $27^{\circ} \mathrm{N}$. The time variation from 1978 to 1993 is shown in Fig. 4. Jupiter's orbital period is approximately 11.8 years; the last northern summer sol-
stice occurred in 1988.7. The influence of seasonally varying insolation is most evident at high latitudes, where more or less
steady warming is evident between 1984 and 1989 during northern hemisphere spring and summer, while southern latitudes cooled during the same period. Seaind with height above the clouds. 
sonal maxima and minima appear within 2 years after the solstice, which is roughly consistent with the radiative time constant of $\sim 6$ $\times 10^{7} \mathrm{~s}(8)$ which implies a lag between the insolation and the thermal response of $\sim 1.5$ year. The amplitude of this seasonal signal is roughly consistent with seasonal models (9), but it is surprising that the seasonal amplitudes in the southern hemisphere are higher than their northern counterparts. Summer solstice in the northern hemisphere occurs very near perihelion, suggesting that the northern hemisphere should be warmer. The annual component of the response to seasonal forcing is an important signal in the temporal behavior of zonal mean temperatures. Nevertheless, it is mixed with several of other variations, which make it difficult even to see the repeating nature of the seasonal cycle for some regions.

Among the variations not related to seasonal insolation variations is that of the EZ which appears to cool by nearly $3 \mathrm{~K}$ between 1978 and 1983. Despite the lower spatial resolution of the ground-based data before 1982, we believe this to correspond to a real temperature decrease, since the Voyager IRIS experiment measured an equatorial temperature decrease of $2 \mathrm{~K}$ at 150 mbar occurring during the 4-month interval separating the Voyager 1 and 2 encounters (7). After 1985, the equatorial temperature undergoes approximately two cycles of a quasi-periodic oscillation with a variable period ranging from 2 to 5 years and an amplitude of $\sim 0.4 \mathrm{~K}$. We believe that this is related to the quasi-periodic oscillation discovered in Jupiter's stratosphere (1), a possible analog of the Earth's quasibiennial oscillation (10). A comparison of the time variation of the equatorial temperature at the $250-\mathrm{mbar}$ and 20 -mbar levels is shown in Fig. 5A (upper panel). The 20-mbar temperatures correspond to a coarse average over a depth of approximately two scale heights between 50 and $1 \mathrm{mbar}$ (1). The oscillations at the two levels remain roughly $180^{\circ}$ out of phase even when the period of oscillation grew noticeably shorter after 1990. Stratospheric and tropospheric temperature oscillations were derived from a two-dimensional (latitude and
Fig. 3. Zonal mean temperature and vertical wind shear as a function of planetocentric latitude and time. Epochs are identified by year and month (see Table 1). The dotted curves surrounding each (solid) zonal mean temperature profile illustrate $\pm 1 \sigma$, which includes contributions from both the noise and the actual longitudinal variations of temperature, with the latter dominating. The vertical dotted lines show the location of zonal wind shear which mark the historical northern and southern boundaries of the NEB and SEB. The large minimum value of the shear, often off the scale, required to illustrate more subtle variations, is (1982) $-42.8,(1983)$ $-64.4,(1984)-75.6,(1985)$ -51.5 , (1986) $-55.8 \mathrm{~m}$ $\mathrm{sec}^{-1}$ (scale height) $^{-1}$. The values of the zonal wind shears within a few degrees of the equator are uncertain owing to the breakdown of the thermal wind equation and the uncertainty in the assignment of latitude. Nevertheless, at least qualitatively, the strong negative shear just south of the equator and its time variability appear to be real.

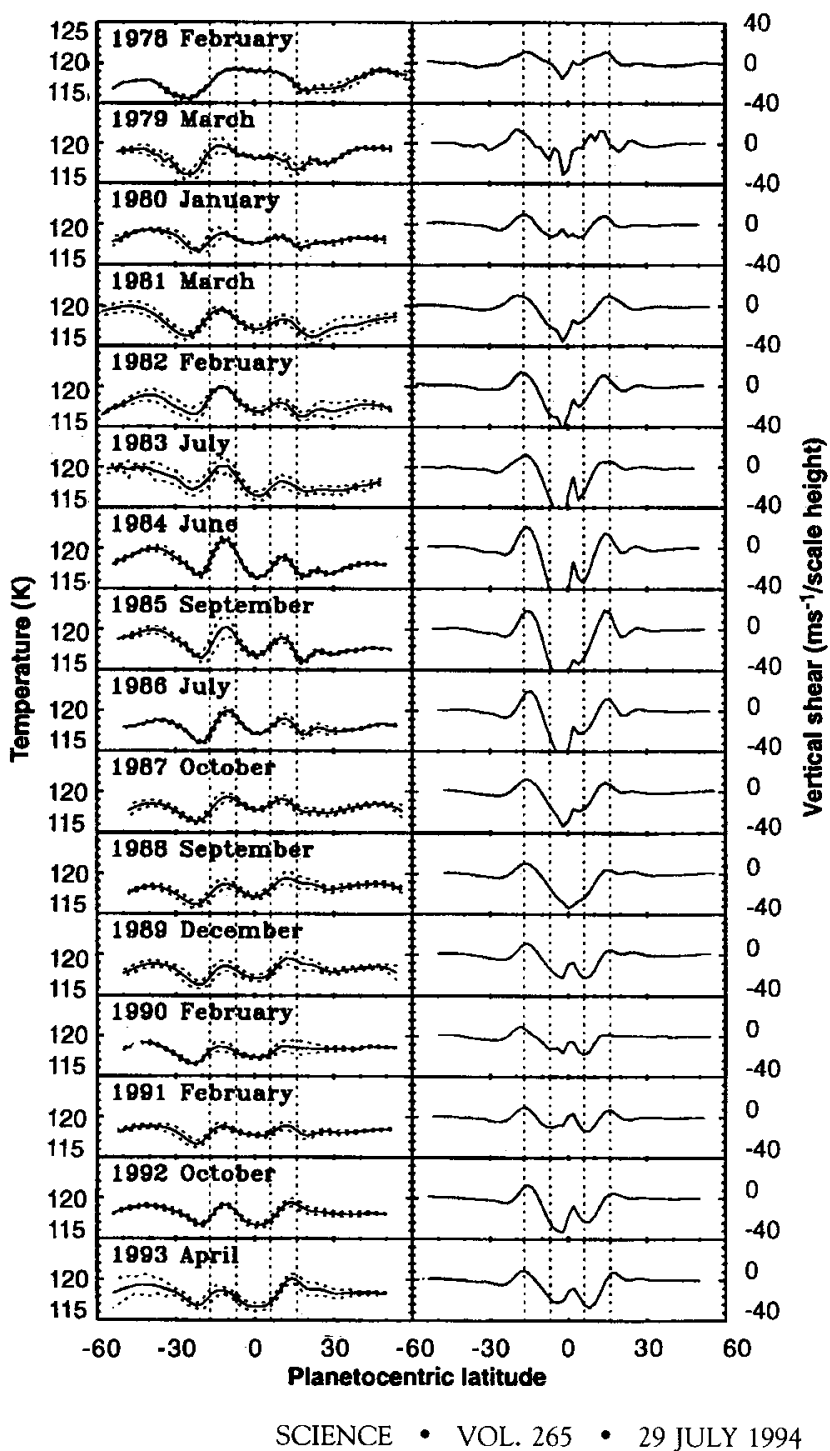

height) dynamical model in which an equatorial temperature oscillation was forced by stresses exerted by vertically propagating, equatorially trapped waves (Fig. 5B) (I1). These calculations were very similar to those performed in similar models of the Earth's quasibiennial oscillation (12). The

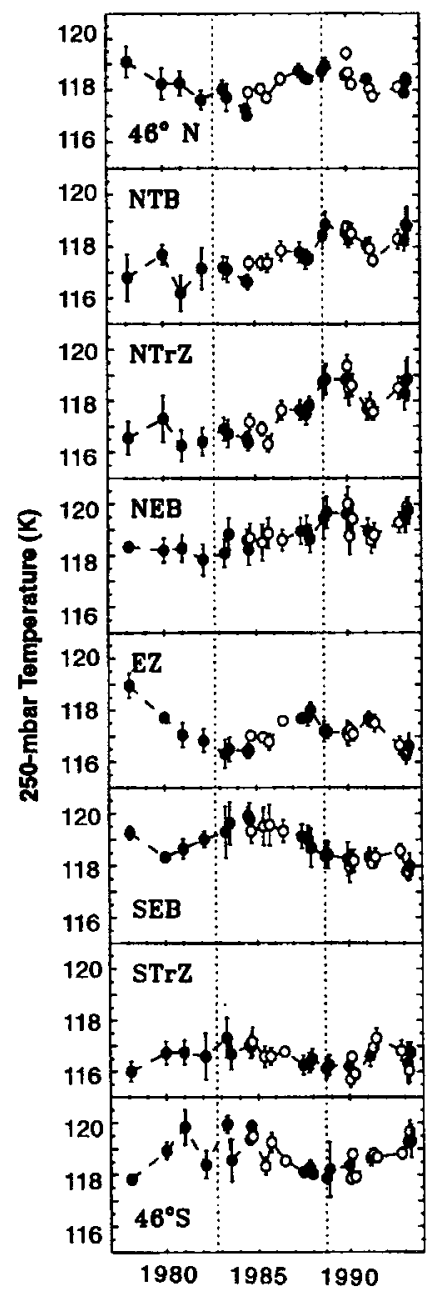

Fig. 4. Zonally averaged 250-mbar temperatures as a function of time for eight different latitudes. Temperatures were averaged in the $2^{\circ}$ latitude bins corresponding to $24^{\circ} \mathrm{N}$ (planetocentric) for the center of the NTB, $20^{\circ} \mathrm{N}$ for the $\mathrm{NTrZ}, 12^{\circ} \mathrm{N}$ for the NEB, $2^{\circ} \mathrm{N}$ for the EZ, $8^{\circ} \mathrm{S}$ for the northern component of the South Equatorial Belt (SEB), and $22^{\circ} \mathrm{S}$ for the STrZ. In addition, temperatures at $46^{\circ} \mathrm{N}$ and $46^{\circ} \mathrm{S}$ are shown. The position of the NTB, determined from a local maximum temperature poleward of the warm NEB, appeared to vary with time (see Fig. 3). Southern solstice at 1982.76 and northern solstice at 1988.69 are marked with dotted lines. Dates of the observations are given in Table 1 . Observations with open symbols denote averages over a single map, that is, over only a $\sim 70^{\circ}$ range of longitude. Vertical bars denote the standard deviation of the temperature mean, arising from both the observational noise and the zonal variability of temperature. For example, the standard deviations in the $E Z$ are much lower than those for the NEB or SEB. 
ratio of the tropospheric to stratospheric oscillation amplitude in the model $(0.7)$ is approximately consistent with the data, but the relative phasing of the signals at the two pressures is different. Comparing the phasing of the oscillations in the model to the data in any detail may not be very meaningful, however, since the model assumes oscillations forced at a single frequency, whereas the data indicate a quasi-periodic oscillation. More observations and modeling are required to determine whether the tropospheric signal is truly related to the stratospheric oscillation, but we propose such a relation as the best explanation we have at present for the behavior of the equatorial tropospheric temperature between 1985 and 1993.

The equatorial coolings observed near 1980 and 1992 (Fig. 4) coincided with periods of visible whitening of the EZ (13 15). These are consistent with a picture of local upwelling during cooling, which brings relatively fresh and bright condensed $\mathrm{NH}_{3}$ to a detectable altitude or which increases the local amount of saturated $\mathrm{NH}_{3}$ gas available for condensation. However, the EZ cooling in late 1988 does not correspond to such a visible change.

Among the most interesting temporal variability seen in the ground-based data occurred within a region bounded on the south by the northern boundary of the NEB $\left(-15^{\circ} \mathrm{N}\right)$ and on the north by the northern boundary of the NTB $\left(\sim 27^{\circ} \mathrm{N}\right)$. In the center of this region lies the strong prograde jet at $20^{\circ} \mathrm{N}$; to north and south lie the retrograde jets associated with the northern boundaries of the belts. The shape of the temperature profiles in this latitude band changed significantly with time, especially during 1984-1990 (Fig. 3). Accompanying the temperature changes in the latitude band between the NEB and NTB is a substantial reduction of the $250-\mathrm{mbar}$ positive vertical zonal wind shear over the retrograde jet at the northern boundary of the NEB and of the negative vertical shear over the strong prograde jet. These vertical shears are likely to be underestimated owing to the $\sim 5^{\circ}$ spatial resolution of the measurements, particularly for the shear over the narrow prograde jet (7). If the zonal wind at the cloud-top level ( $\sim 600$ to 700 mbar) is relatively steady (16), the acceleration at the 250-mbar level of the mean zonal wind over the retrograde jet implied by the change in the vertical shear amounts to at least $-3 \mathrm{~m} \mathrm{sec}^{-1}$ per terrestrial year.

The qualitative sense of the acceleration over the prograde and retrograde jets suggests that they underwent a transition from a state of general decay with altitude above the cloud-top level to a state in which they were nearly constant with height. Presumably, it is the result of convergence of the meridional or vertical flux of momentum transported by turbulent eddies in the region, and hence its magnitude and sign may provide clues to the nature of the forcing such eddies exert on the zonal mean flow. If eddies transporting momentum horizontally were absorbed within the width of the retrograde jet $\left(\sim 5^{\circ}\right)$, then a horizontal wave stress $\overline{\rho u^{\prime} v^{\prime}}$ of order, of $0.04 \mathrm{~N} \mathrm{~m}^{-2}$ is implied (where $\rho$ is the density, $u^{\prime}$ and $v^{\prime}$ denote departures of the local eastward and northward wind from their zonal averages, and the overbar denotes zonal averaging). This stress is smaller by about two orders of magnitude than estimates for the rate that kinetic energy is fed into the zonal jets at the cloud-top level (at about $500 \mathrm{mbar}$ ), which are on the order of $2 \mathrm{~N} \mathrm{~m}^{-2}(17)$. If instead a vertical eddy momentum flux were absorbed over a depth of about one scale height, centered at the 250-mbar level, the implied eddy stress would be $\overline{\rho u^{\prime} w^{\prime}} \sim 10^{-4} \mathrm{~N} \mathrm{~m}^{-2}$, where $w^{\prime}$ is the departure of the vertical velocity from its zonal average. This number is of the same order of magnitude as estimates derived for the vertical stress of equatorially trapped waves at Jupiter's equator (10) and of waves at high latitudes (18), and therefore may indicate the general order of magnitude of wave momentum carried upward by vertically propagating waves generated by Jupiter's convection. The true nature of the mechanism that caused the observed changes in the vertical wind shears cannot be ascertained without detailed observations of the eddy winds and temperatures.

It is also interesting to note changes of the visible appearance of these regions which were concomitant with the thermal changes. The visually dark NTB brightens periodically $(19,20)$ to the same level as the nearby North Tropical Zone (NTrZ). It brightened in the middle of 1987 and then darkened in 1990 during a major outbreak of white and dark spots in its southern component $(14,21-23)$. The 1987-1990 warming (Fig. 4) may be associated with this brightening $(13,20,22,23)$. The visually dark NEB also broadened to the north in early 1988, and then receded during 1989, leaving an array of dark barges and bright ovals (14, $24,25)$. This broadening coincided with a pronounced warming in the NEB and the latitude labeled as the NTrZ in Fig. 4. The South Tropical Zone (STrZ) warming in 1993 (Figs. 3 and 4) corresponded to the most extensive darkening of this zone (15). The NEB broadening (or NTrZ darkening) and the STrZ darkening are consistent with rapid adiabatic warming with subsidence and resublimation of condensed (and visibly bright) $\mathrm{NH}_{3}$ ice particles to reveal darker

Fig. 5. Comparison of the time behavior of the equatorial zonal mean temperature at the 250-mbar and 20mbar levels. Only the deviation of temperatures from their time average is shown. (A) The 250-mbar temperature from this study (solid line) is compared with the 20-mbar temperature derived by Orton et al. (1) (dashed line). (B) Time behavior of zonal mean temperatures at the 250 -mbar (solid line) and 20-mbar (dashed line) levels, calculated with a two-dimensional (latitude-height) dynamical model (11). Equatorial oscillations of zonal mean wind and temperature in the model were forced by a vertically propagating, eastward propagating kelvin wave and an idealized, westward propagating "anti-kelvin" wave (10). Both modes corresponded to wave number 1 and had phase speeds of $\pm 120 \mathrm{~m} \mathrm{sec}^{-1}$. The model calculation shown produced a single oscillation period of 6 years, while the data appear to indicate quasi-periodic behavior with dominant periods ranging between roughly 2 and 6 years.
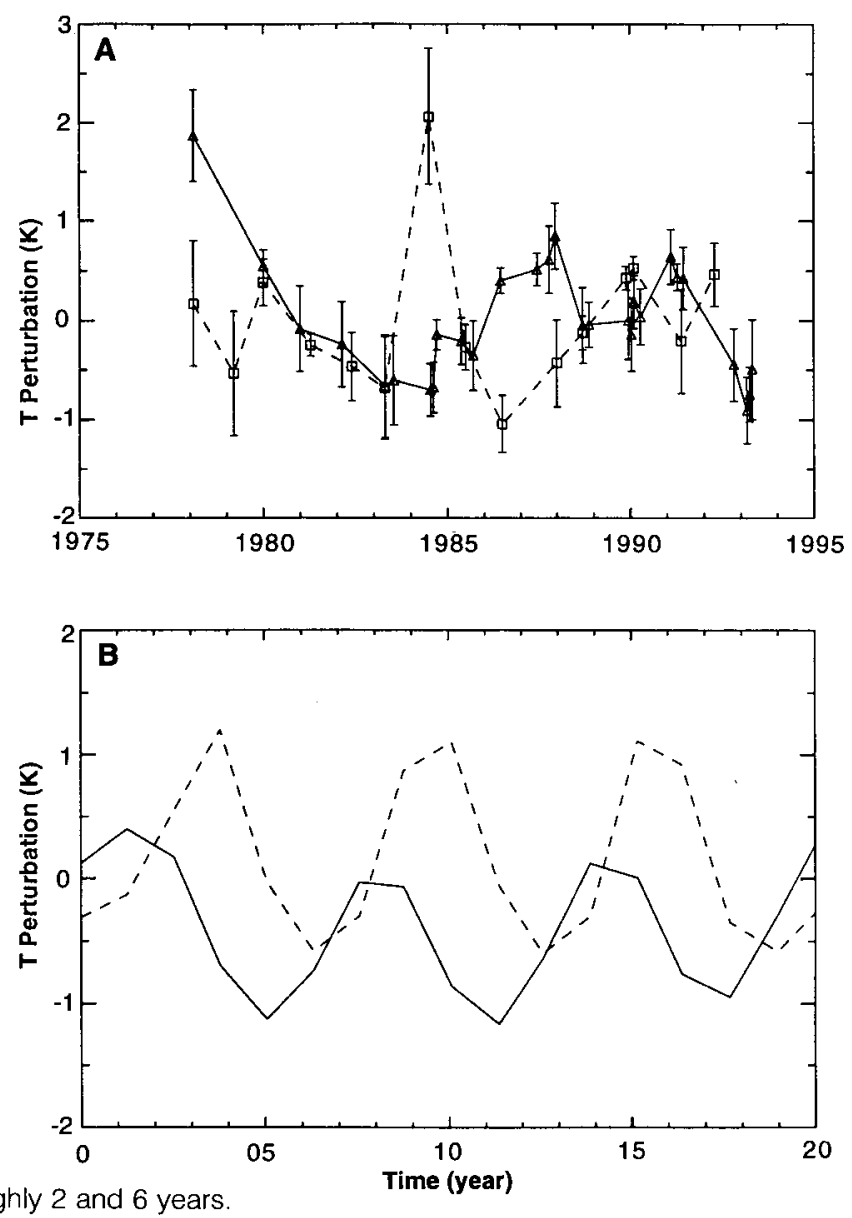
particles below or darker nucleation sites. The coincidence of the visible brightening of the NTB and its warming or merger with the NEB is not so easily understood. However, some axisymmetric regions whose visible clouds were quite variable exhibited little correlated temperature variability. For example, there were no thermal variations of the STrZ before 1991 during the development of several discrete columns and streaks $(13,26,27)$. Another surprise was that the $\mathrm{SEB}$, which has undergone several dramatic albedo changes at all longitudes between 1989 and $1993(14,15,25)$, showed no contemporaneous change of its temperature

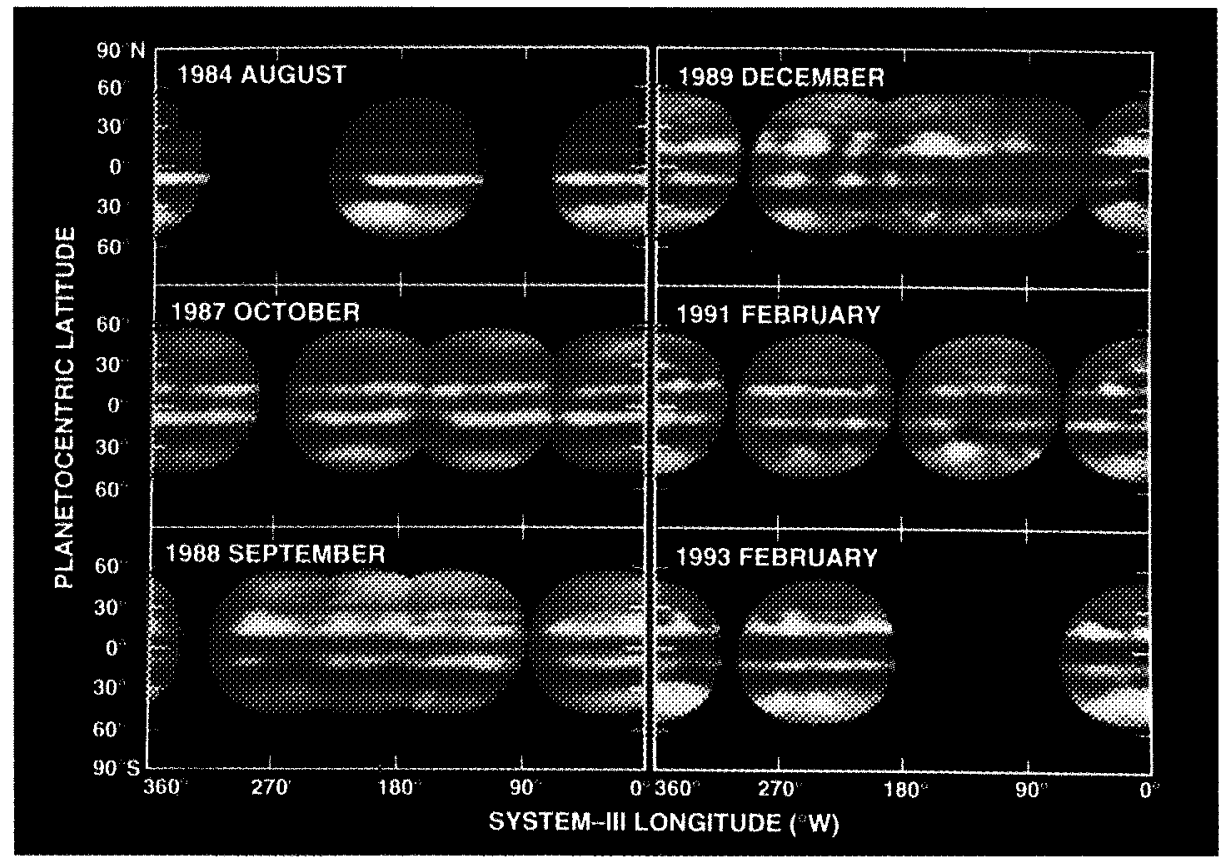

Fig. 6. Cylindrical projections of temperature profiles derived from selected groups of raster-scan images of Jupiter taken over several sequential days between 1984 and 1993 (see Table 1). The grid scale is $2^{\circ}$ in latitude and longitude. Unusually large excursions from the average near the limbs and "seams" between overlapping maps are the result of the inconsistency between the actual temperature profile and the assumed form of the temperature profiles (Fig. 2). The temperature scale for all the maps is $112.0-121.5 \mathrm{~K}$, except for 1984 August which is $113.0-122.5 \mathrm{~K}$.

Fig. 7. Plots of stratospheric temperature (A, 20-mbar pressure) and tropospheric temperature (B, 250-mbar pressure), for 1988 September 26-28 and November $30-$ December 2 , as it varies with System III longitude. The tropospheric temperature profiles correspond to $13^{\circ} \mathrm{N}$ latitude (planetocentric), while the stratospheric temperatures correspond to $22^{\circ} \mathrm{N}$. The 1988 November temperatures for both stratosphere and troposphere are plotted with a longitudinal offset of $-24^{\circ}$ in order to illustrate the strong correla-

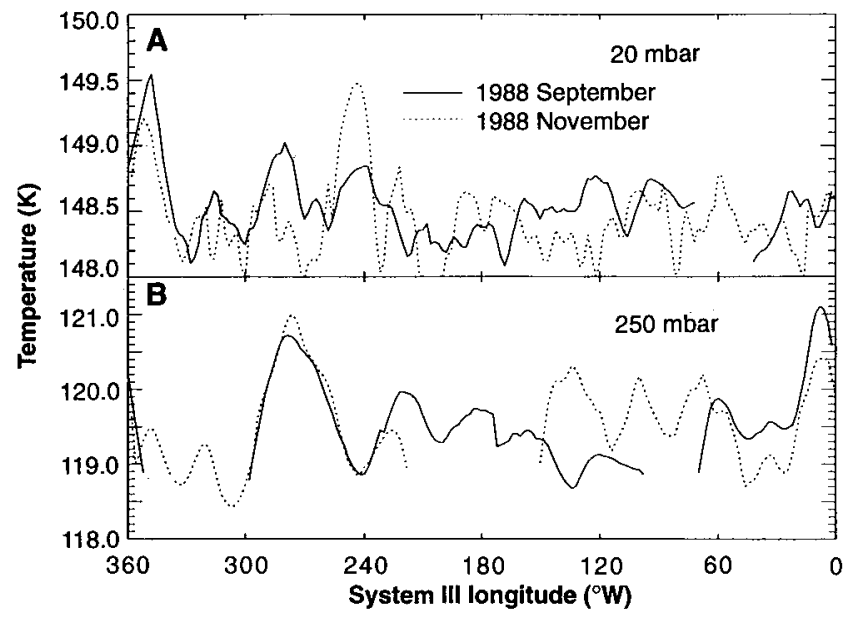
tion of individual features in both pressure levels with this offset.

structure which is easily separable from the seasonal signature.

Longitudinal structure. Maps of the 250mbar temperature field which are the most complete in longitudinal coverage are shown in Fig. 6. The most prominent feature in which appears as a very cold Spot (GRS) in each of the maps, subtending $-10^{\circ}$ in latitude and $18^{\circ}$ in longitude, commensurate with its visible appearance. The $250-\mathrm{mbar}$ temperature of the GRS is $115.3 \pm 0.4 \mathrm{~K}$, meking it the coldest region on the planet between $60^{\circ} \mathrm{S}$ and $60^{\circ} \mathrm{N}$ latitude. It is about $1.3 \pm 0.5 \mathrm{~K}$ colder than the relatively cold 
identification is more evident in the $\mathrm{No}$ vember data. If we accept the premise that the tropospheric and stratospheric waves correspond to a single disturbance, then the identifications made above imply a variation of wave phase with altitude, suggesting that the disturbance is vertically propagating, rather than exponential, in nature.

Since the thermal waves observed in September and November 1988 are composed predominantly of zonal wavelengths of order 40,000 to $70,000 \mathrm{~km}$, and since their amplitudes suggest that the Rossby number associated with their motion is small, it is likely that their propagation is governed by the dispersion relation for linear, planetary scale Rossby waves. Such waves can propagate vertically only when their phase speed, measured relative to the local zonal mean wind, is opposite in sign to the local latitudinal gradient of potential vorticity. If the effect of vertical stratification of the atmosphere on the potential vorticity $q$ is negligible, then the latitudinal gradient of $q$ is given approximately by

\section{$(1 / a) \partial q / \partial \phi \simeq(1 / a) \partial f / \partial \phi-\left(1 / a^{2}\right) \partial^{2} \pi / \partial \phi^{2}$}

In the retrograde jet at $15^{\circ} \mathrm{N}$, which has an amplitude of $-30 \mathrm{~m} \mathrm{sec}^{-1}$ and a width of $\sim 4^{\circ}$ of latitude, $(1 / a) \partial q / \partial \phi$ is negative (24), and as a consequence, vertical propagation occurs for waves with phase speeds that are eastward relative to the mean zonal flow. Our deduction that the thermal waves observed at $13^{\circ} \mathrm{N}$ are vertically propagating is consistent with this result; their phase speed of $-5.5 \mathrm{~m} \mathrm{sec}^{-1}$ relative to system III is eastward with respect to the zonal wind over most of the retrograde jet. (Actually, we have deduced the zonal group velocity rather than the phase speed from the longitudinal offset shown in Fig. 7, but the two velocities are very nearly equal for the case of Rossby waves with zonal wavelengths much longer than the deformation radius, which is of order $1000 \mathrm{~km}$ or less). Such waves are expected to be latitudinally trapped in a waveguide within the jet between the critical surfaces at which the zonal wind matches the phase speed. Normally, the wave propagation region would also be bounded above by a critical layer because the vertical shear is positive (eastward) above the retrograde jet, but during times of weak vertical shear, the waves might be able to penetrate to high altitudes. Such a period of weak vertical shear existed during September and November of 1988. If this interpretation is correct, we should expect to see a correlation between periods of high stratospheric wave activity and periods of reduced vertical shears over the retrograde jets associated with the NEB and $\mathrm{SEB}$, a view which is generally consistent with the available data but in need of more rigorous testing.
We also examined the data for the presence of "slowly moving thermal features", such as those reported in the Voyager IRIS data (29). Correlation coefficients were computed by normalizing to the longitude extent covered by both maps under consideration, with offsets reported with respect to System III. The presence of significant correlations (in Table 2) is evidence for such features. However, with few exceptions, they were found only after 1986 and only for those latitudes with the highest amplitude waves, that is, the NEB and SEB. For the $\mathrm{SEB}$, this correlation is always dominated by the cold GRS when it is detectable and the phase velocity corresponding to these offsets divided by the difference in observation time is in the range of -4 to $-5 \mathrm{~m} \mathrm{sec}^{-1}$. For the NEB, this value of the phase velocity ranges between -2 and $-12 \mathrm{~m} \mathrm{sec}^{-1}$. We suspect that most of this variability is real. For 1989 December through early 1990, some wave structure is easily detectable at the equator. A comparison between the 1990 January and 1990 February single-image maps shows a significant equatorial correlation midway between the NEB and the SEB values, although we found no significant correlation at the equator between the 1989 December maps and either 1990 January or February maps.

Deming et al. (30) detected wave number 10 features covering the entire lowlatitude region of the planet. Our data revealed no features of this type, particularly any covering a broad range of latitudes (except possibly in December 1989, as shown in Fig. 6), although we saw some evidence for slowly moving thermal features, as described above. One possible reason is that Deming et al. observed in a

Table 2. Drift rates of 250-mbar temperature waves.

\begin{tabular}{|c|c|c|c|c|c|}
\hline \multicolumn{2}{|c|}{ Epoch } & \multirow{2}{*}{$\begin{array}{l}\text { Planetocentric } \\
\text { latitude }\end{array}$} & \multicolumn{2}{|c|}{ Delta } & \multirow{2}{*}{$\begin{array}{l}\text { Drift rate } \\
(\mathrm{m} / \mathrm{s})\end{array}$} \\
\hline Start & End & & $\begin{array}{l}\text { Longitude } \\
\qquad(\mathrm{W})\end{array}$ & $\begin{array}{l}\text { Time } \\
\text { (days) }\end{array}$ & \\
\hline 1987 October & 1987 December & $\begin{array}{l}-10 \\
+14\end{array}$ & $\begin{array}{l}-20 \pm 10 \\
-12 \pm 6\end{array}$ & 64 & $\begin{array}{l}-4.3 \pm 2.1 \\
-2.6 \pm 1.3\end{array}$ \\
\hline 1988 September & 1988 December & $\begin{array}{l}-20 \\
-12 \\
+12 \\
+18\end{array}$ & $\begin{array}{ll}-20 \pm & 4 \\
-16 \pm & 2 \\
-20 \pm & 2 \\
-22 \pm & 2\end{array}$ & 66 & $\begin{array}{l}-4.4 \pm 0.8 \\
-3.4 \pm 0.4 \\
-4.2 \pm 0.4 \\
-4.5 \pm 0.5\end{array}$ \\
\hline 1989 December & 1990 January & $\begin{array}{l}-10 \\
+14 \\
+22\end{array}$ & $\begin{array}{r}-30 \pm 10 \\
-4 \pm 4 \\
-10 \pm 4\end{array}$ & 21 & $\begin{array}{r}-19.9 \pm 6.6 \\
-2.6 \pm 2.6 \\
-6.2 \pm 2.5\end{array}$ \\
\hline 1990 January & 1990 February & $\begin{array}{r}-10 \\
+2 \\
+14\end{array}$ & $\begin{array}{l}-30 \pm 2 \\
-28 \pm 2 \\
-20 \pm 2\end{array}$ & 33 & $\begin{aligned}-12.7 & \pm 0.8 \\
-11.8 & \pm 1.7 \\
-8.3 & \pm 0.8\end{aligned}$ \\
\hline 1991 February & 1991 April & $\begin{array}{l}+14 \\
+28\end{array}$ & $\begin{array}{l}-14 \pm 2 \\
-12 \pm 2\end{array}$ & 51 & $\begin{array}{l}-3.8 \pm 0.5 \\
-2.9 \pm 0.5\end{array}$ \\
\hline 1993 February & 1993 March & $\begin{array}{l}-20 \\
-10 \\
+14\end{array}$ & $\begin{array}{r}-10 \pm 2 \\
-6 \pm 4 \\
-20 \pm 6\end{array}$ & 29 & $\begin{array}{l}-4.6 \pm 0.9 \\
-2.9 \pm 1.9 \\
-9.5 \pm 2.8\end{array}$ \\
\hline 1993 March & 1993 April & $\begin{array}{l}-20 \\
+14 \\
+24\end{array}$ & $\begin{array}{l}-10 \pm 2 \\
-24 \pm 4 \\
-24 \pm 4\end{array}$ & 28 & $\begin{array}{r}-4.8 \pm 1.0 \\
-11.1 \pm 2.0 \\
-11.1 \pm 2.0\end{array}$ \\
\hline
\end{tabular}

SCIENCE • VOL. 265 • 29 JULY 1994 spectral region which was also sensitive to variations of the $\mathrm{NH}_{3}$ gas and ice distribution, as well as to variations of temperature. Furthermore, the amplitude of their waves was somewhat smaller than the characteristic noise level of most of our data.

Remaining questions. Foremost among our remaining questions concerns the relation between changes in zonal mean temperature and visual albedo in major axisymmetric regions. The coincidence of a thermal warming with a decrease of albedo in mechanism where warming of the region led to resublimation of visibly bright $\mathrm{NH}_{3}$ ice particles, releaving darker particles at greater depths or leaving behind dark nucleation cores. On the other hand, warming of the NTB during 1987 to 1990 was accompanied by an increase in the visual albedo, while albedo changes in the SEB were not accompanied by significant temperature changes, at least at the same time. Part of our further inquiry into this matter will be to analyze the physical properties of clouds from our own infrared database, together with a description of the distribution of $\mathrm{NH}_{3}$ gas and tropospheric temperatures at pressures greater than 250 mbar. Another approach to this question is to examine the correlation between longitudinal variability of clouds and temperature, which we addressed here only for the axisymmetric regions and the GRS. between temporal thermal variations that arise from seasonal radiative forcing rather than other effects. Modeling the influence of seasonal radiative forcing on temperatures in some detail will allow us to separate these the NTrZ during 1988 is consistent with a

We also have not fully differentiated 
influences and, among other things, to determine the origin of the hemispherical asymmetries in the temperature field. Other questions might be answered by a combination of dynamical models and continued observations through at least another seasonal cycle, particularly in view of the potential for improved data quality. Several such questions are associated with zonal wave structure. Our results indicate upward propagation of tropospheric waves to the stratosphere in late 1988 (Figs. 7 and 8), but other zonal waves had no apparent influence on the stratosphere. Did the 1988 waves propagate upward solely because they coincided with a weak vertical shear over the NEB? Do the regular wave structures observed by Deming et al. (30) extend to the temperature field alone? There is clearly some indication of slowly thermal waves in the NEB and SEB; what determines the dominant wavelengths and phase speeds of these waves? Understanding wave behavior at lower latitudes may be very significant in studying the Galileo probe results for the temperature profile near $7^{\circ} \mathrm{N}$ latitude.

The vast improvements in efficiency of recent technological developments in middle-infrared array detectors compared to raster-scanning and middle-infrared cameras, should provide greater coverage of Jupiter over a broader spectral range, characterized by higher signal-to-noise ratios with much better prospects for absolute flux calibration. Although angular resolution in the $18-\mu \mathrm{m}$ region is diffraction-limited at the IRTF, observations with larger telescopes or application of image restoration techniques may improve the effective spatial resolution, enabling us to study smaller features in the size regime where numerous visible and near infrared features illustrate the full variety of Jupiter's diverse mesoscale weather.

\section{REFERENCES AND NOTES}

1. G. S. Orton et al., Science 252, 537 (1991).

2. Pioneer 10 and 11 infrared results have been summarized [A. P. Ingersoll, G. Münch, G. Neugebauer, G. S. Orton, in Jupiter, T. Gehrels, Ed. (Univ. Arizo- na Press, Tucson, 1976), pp. 197-205; G. S Orton, A. P. Ingersoll, in ibid., pp. 206-215]. Recent Voyager 1 and 2 infrared results of relevance are found in F. M. Flasar et al., $J$ Geophys. Res. 86, 8759 (1981); B. J. Conrath, F M. Flasar, J. A. Pirraglia, P. J. Gierasch, G. E. Hunt, ibid., p. 8769; and P. J. Gierasch, B. J. Conrath, J. A. Magalhăes, Icarus 67, 456 (1986).

3. Before 1987 , observations were made with a filter centered at $17.8 \mu \mathrm{m}$ in wavelength. These observations have been scaled to agree with the absolute radiances of the $18-\mu \mathrm{m}$ filter, as there is an insignificant difference in the atmospheric level sounded and in the characteristic center-to-limb behavior of the radiance. Therefore, we use the $18-\mu \mathrm{m}$ designation to refer to the suite of data covering observations from 1980 to 1993 , including those made with the $17.8-\mu \mathrm{m}$ filter.

4. The model of $W$. Meyer, $L$. Frommhold, and $G$. Birnbaum [Phys. Rev. 39, 2434 (1989)] was used for $\mathrm{H}_{2}-\mathrm{H}_{2}$ collision-induced absorption and that of J. Borysow, L. Frommhold, and G. Birnbaum [Astrophys, J. 326, 509 (1987)] was used for $\mathrm{H}_{2}$-He. The variation of para- compared to ortho$\mathrm{H}_{2}$ as a function of latitude was modeled after that derived from the voyager IRIS observations by B. J. Conrath and P. J. Gierasch [/carus 57, 184 (1984)].

5. G. F. Lindal et al., J. Gecphys. Res. 86, 8721 (1981)

6. W. M. Sinton, W. W. Macy, G. S. Orton, lcarus 42, 86 (1980)

7. P. J. Gierasch, B. J. Conrath, J. A. Magalhaes, ibid. 67, 456 (1986)

8. F. M. Flasar, in Time-variable Phenomena in the Jovian System, M. J. S. Belton, R. A. West, J. Rahe, Eds. (NASA Special Publication 494, NASA, Washington, DC, 1989), pp. 324-343

9. C. Bezanger, B. Bézard, D. Gautier, in The Jovian Atmospheres. M. A. Allison and L. D. Travis, Eds. (NASA Conference Publication 2441, NASA Washington, DC, 1986), pp. 79-81; Jupiter's (sidereal) orbital period is 11.86 years.

10. C. B. Leovy, A. J. Friedson, G. S. Orton, Nature 354,380 (1991).

11. A. J. Friedson, Bull. Am. Astron. Soc. 24, 1038 (1992).

12. T. Dunkerton, J. Atmos. Sci. 48, 236 (1991)

13. J. H. Rogers, Ed., Mem. Brit. Astron. Assoc. 43, No. 3 (1992).

14. J. H. Rogers, J. Brit. Astron. Assoc 102, 135 (1992).

15. _ ibid., p. 324.

16. R. F. Beebe, L. F. Huber, N. J. Chanover, Bull. Am. Astron. Soc. 24, 1038 (1992)

17. A. P. Ingersoll et al., J. Geophys. Res. 86, 8733 (1981).

18. R. A. West, A. J. Friedson, J. F. Appleby, Icarus 100,245 (1992)

19. R. F. Beebe, G. S. Onton, R. A. West, in TimeVariable Phenomena in the Jovian System, M. J. S. Belton, R. A. West, J. Rahe, Eds. (NASA Special Publication 494, NASA, Washington, DC, 1989), p. 245
20. A. Sanchez-Lavega and J. A. Quesada, Icarus 76 $533(1988)$

21. A. Sanchez-Lavega, in Time-Variable Phenomena in the Jovian System M. J S Belton, R. A. West, J. Rahe, Eds. (NASA Special Publication 494 NASA, Washington, DC, 1989), p. 306.

22. I. Miyazaki, D. Parker, P. Laques, J. Lecacheux, Icarus 94, 92 (1991).

23. T. Little, Bull. Am. Astron. Soc. 21, 945 (1989).

24. J. H. Rogers, J. Brit. Astron. Assoc. 101, 22 (1991).

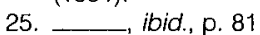

26. A. Sanchez-Lavega and R. Rodrigo, Astron. Astrophys. 148, 67 (1985).

27. J. Rogers, J. Brit. Astron. Assoc. 97, 46 (1986) ibid. 98, 151 (1988).

28. T. Satoh and K. Kawabata, Astrophys. J. 384 298 (1992); D. M. Kuehn and R. F. Beebe, lcarus 101, 282 (1993); P. Yanamandra-Fisher, G. Orton, J. Friedson, Bull. Am. Astron. Soc. 24, 1039 (1992); J. Rogers, J. Br. Astron. Assoc. 103, 157 (1993)

29. J. A. Magalhaes, A. L. Weir, B. J. Conrath, P. J. Gierasch, S. S. Leroy, Nature 337, 444 (1989).

30. D. Deming et al., Astrophys. J. 343, 456 (1989).

31. We thank the Jet Propulsion Laboratory Supercomputing Project for help in establishing several sets of atmospheric models from which the tables of radiance as a function of temperature were derived; the staff and management of the Infrared Telescope Facility for their support, particularly E. Becklin, the former acting IRTF division chief and R. Joseph, current division chie for their efforts to schedule a monthly monitoring program; the IRTF telescope allocation committee for their long-term support for this research; C. Russell, who was supported by California Institute of Technology Summer Undergraduate Research Fellowship at the Jet Propulsion Laboratory for programming help; J. Klavetter for assistance with observing and data reduction during 1982; and J. Rogers of the British Astronomical Association for references to the continuous record of visible observations of Jupiter provided by the active nonprofessional commu nity. This research was sponsored by the National Aeronautics and Space Administration for work performed at the Jet Propulsion Laboratory, California Institute of Technology, and at the Institute for Astronomy, University of Hawaii; by the National Research Council of Canada for work performed at York University: Galileo Project (G.S.O.) for this portion of his pre-encounte interdisciplinary investigation. These data were collected as one element of a larger set of observations coordinated by the International Jupiter Watch. G.S.O., P.A.Y.-F, A.J.F., J.C., H.B.H., K.H.B., J.T.B., T.Z.M., R.A.W., G.J.V., D.K.L., and R.R. were visiting astronomers at the Infrared Telescope Facility which is operated by the University of Hawaii under contract to NASA

27 December 1993; accepted 2 May 1994 